\title{
Management of air bubble within the excluded aneurysm sac in the early period after endovascular management of abdominal aortic aneurysm
}

\author{
Murat Çelik ${ }^{1}$, Uygar Çağdaş Yüksel ${ }^{1}$, Uğur Bozlar ${ }^{2}$ \\ (1) University of Health Sciences, Gulhane Research and Training Hospital, Department of Cardiology, Ankara, \\ Turkey \\ (2) University of Health Sciences, Gulhane Research and Training Hospital, Department of Radiology, Ankara, Turkey
}

Date submitted:

Sep 27, 2018

Date accepted:

Oct 11, 2018

Online publication date:

March 15, 2019

\section{Corresponding Author: \\ Murat Çelik \\ Gulhane Research and Training \\ Hospital, Cardiology, Ankara, \\ Turkey \\ drcelik00@hotmail.com}

\begin{abstract}
Although technological improvements have increased, EVAR is still not free of complications during or after EVAR-procedure. Infection complication during EVAR is considered quite rare, and contamination during the procedure is proposed the foremost reason. Radiological evidence such as the presence of air bubble within the excluded aneurysmal sac in the early post-procedure period of endovascular aneurysm repair (EVAR) might be an important finding of infection, even in the absence of any symptoms; and thereby, it should be monitored closely.
\end{abstract}

Keywords: Endovascular aneurysm repair, air bubble within the excluded aneurysmal sac, early post-procedure period.

\section{Introduction}

Endovascular aneurysm repair (EVAR) today is rapidly growing as a less invasive and effective treatment approach for patients with abdominal aortic aneurysms (AAA) and suitable anatomies. Although technological improvements have increased, EVAR is still not free of complications and complication rates can be as high as $41 \%$ (1). The most common complication is endoleak. Nevertheless, non-endoleak complications are comparatively uncommon and generally include rupture of the access site arteries, systemic complications, clot embolization and/or ischemic complications, infection complications of the stent graft and/or excluded aneurysmal sac (2). Infection complication during EVAR is considered quite rare, and contamination during the procedure is proposed the foremost reason.

\section{Presentation of Case}

74-year-old male, diagnosed with coronary artery disease was referred to our department with an abdominal pulsatile mass. He was heavy smoker and had hypertension. Computed tomography $(\mathrm{CT})$ imaging revealed an AAA with a maximum diameter of $56 \times 56 \mathrm{~mm}$, involving a segment of approximately $75 \mathrm{~mm}$ at infra-renal level. After discussion with the surgical team, we decided to perform EVAR. Under local anesthesia with mild sedation, bilateral transfemoral access was obtained. The Medtronic Endurant II bifurcated stent graft system was inserted. The main body (ETBF 2813C166EE) was delivered via the right groin and the contralateral limb (ETLW 1616C124EE) through the left groin. Operative time was 55 minutes and 145 $\mathrm{mL}$ contrast was used. We completed the procedure successfully having seen no endoleak on control angiography. Both groin vascular access sites was successfully closed using the Abbott Perclose Proglide suture mediated closure system. The postoperative course was uneventful, and the patient was discharged well on the third post-operative day.

After 3 days of discharge, the patient presented with a complaint of abdominal pain. His physical examination was within normal limits. An abdominal control CT study again performed 
and revealed there was no endoleak. Interestingly, when we examined the CT in more detail, we detected an air bubble in the excluded aneurysmal sac, outside the endograft. Multiplanar reformatted (MPR) images showed that the dimension of air bubble was $14.7 \times 7.11 \mathrm{~mm}\left(72.31 \mathrm{~mm}^{2}\right)$ (Figure 1A).
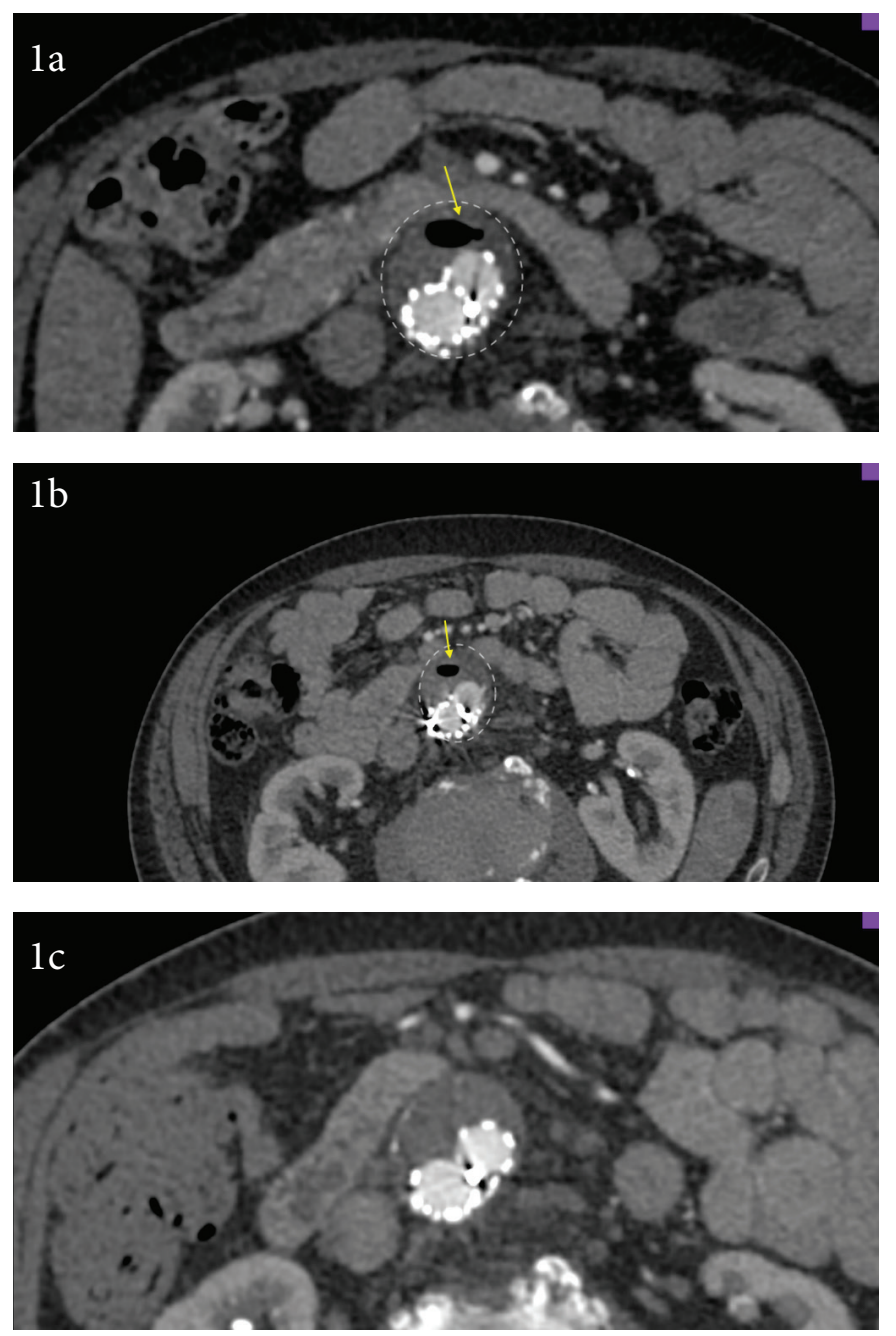

Figure 1. Computed tomography imagings. At the 3rd day of discharge (A), at the 10th day of discharge (B) and at the 17th day of discharge (C). White dashedlines show outer border of excluded aneurysm sac, yellow arrows show the air bubble within the excluded aneurysm sac. Stent struts are clearly seen.

From the point of view of detection of air is an important radiological finding of infection; we questioned the signs and symptoms of infection in the patient. Although the patient was doing well without signs and symptoms of infection, we took blood samples for biochemical assays and blood culture. Laboratory investigations revealed an elevated white blood cell count $(15.2 \times 103$ cells/uL), erythrocyte sedimentation rate (65 $\mathrm{mm} / \mathrm{h})$, high sensitive CRP (170.6 mg/L), and a procalcitonin level $(0.11 \mathrm{ng} / \mathrm{mL})$. We excluded other possible causes of infections. After the consultation of infectious diseases, he was started on broad-spectrum antibiotic therapy with IV 2 grams ceftriaxone doses twice a day with a probable diagnosis of stent graft infection. Control CT scan performed on the 7th day of antibiotic therapy revealed that the sizes of the air bubble had reduced to $8.92 \times 5.04 \mathrm{~mm}\left(40.61 \mathrm{~mm}^{2}\right)$ (Figure 1B). Seven days after IV antibiotic therapy, the patient's abdominal pain completely resolved and the biochemical markers of infection
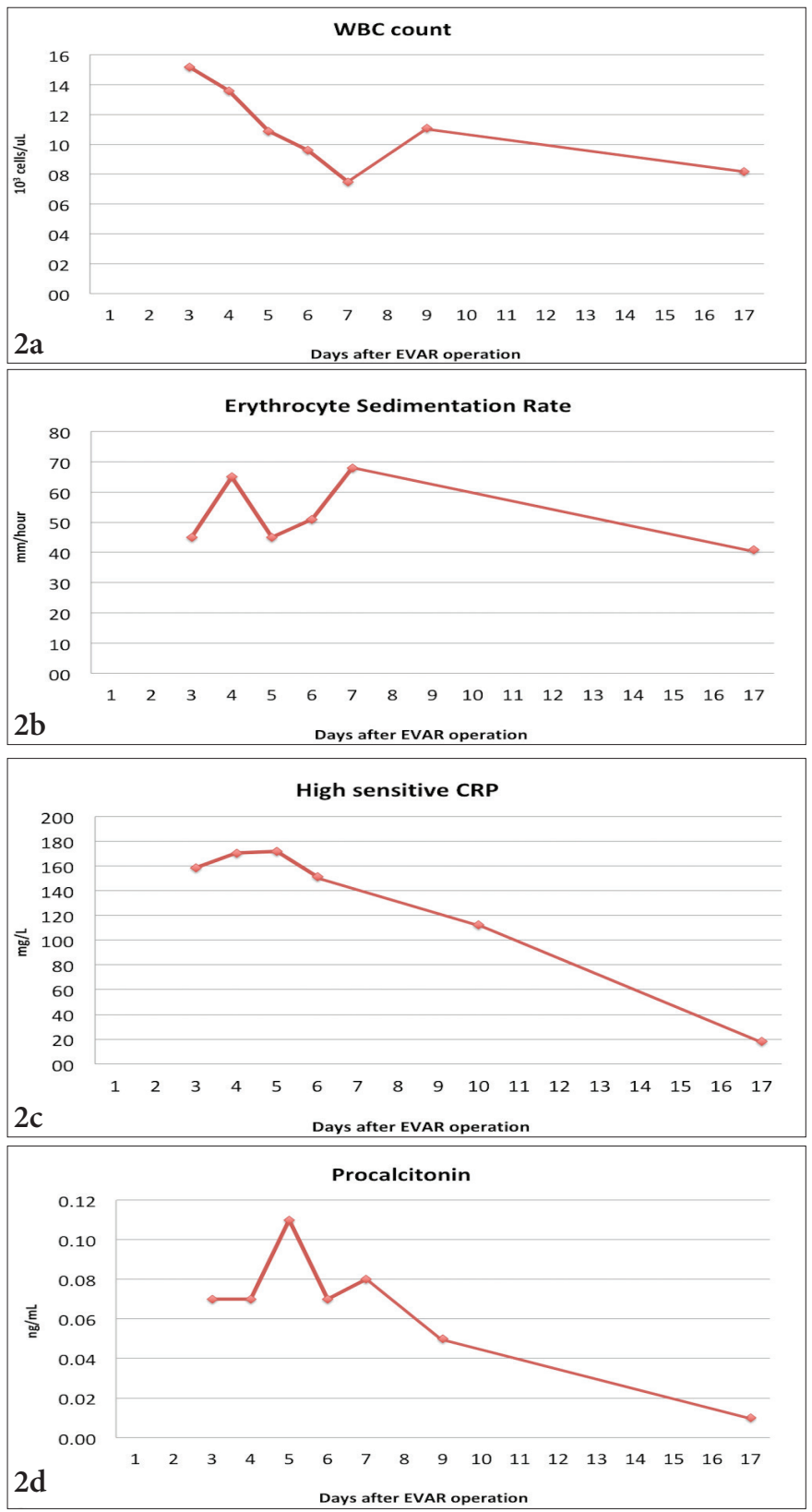

Figure 2. CInfection markers during follow-up. White blood cell count (A), erythrocyte sedimentation rate (B), high sensitive C-reactive protein (C), and procalcitonin level (D).

were significantly decreased. Also, blood culture was negative. Thereby, the patient was discharged on oral antibiotic therapy with ciprofloxacin and metronidazole at recommended dosage. Ten days after oral antibiotic therapy (17 days after diagnosis), control CT revealed the completely disappearing of air bubble (Figure 1C) and the infectious markers were improved in great measure (Figure 2A-D). At the second month of follow-up after discharge, the patient was very good without any complaints and the infection markers were normal.

\section{Discussion}

As with any vascular procedure, EVAR has its own unique set of complications related to several potential mechanisms. Since these complications potentially can be serious, a swift and meticulous radiological diagnosis and often subsequent 
interventional or surgical treatment are required (3). Early complications mostly include procedural causes, whereas delayed complications are mostly systemic complications noted upon follow-up. Graft infection during EVAR of abdominal aorta is considered quite rare with an incidence of $0.4-3.0 \%$ (4), and is associated with high mortality rates that range from $25 \%$ to $50 \%$, if left undiagnosed and/or untreated (5).

Although graft infection usually occurs within the first four months after graft implantation, it is not expected to be seen in a few days after the procedure. Early endograft infection may result from intraprocedural contamination, while late-period infections may result from a remote site infection leading to colonization of the endograft (6). In our case, we observed signs and symptoms of infection 3 days after EVAR procedure.

Diagnosis of infection of endograft, and/or excluded aneurysmal sac is based on clinical and radiological findings. Leukocytosis, fever, and back pain are typical clinical signs. Radiological findings of endograft infection include perigraft fluid, perigraft soft-tissue attenuation, ectopic gas, pseudoaneurysm, focal bowel wall thickening, and hydronephrosis (6). Additionally, the presence of air bubbles within the aneurysmal sac might be a strong indicator of endograft infection. In contrast, some publications stated that perigraft air within the excluded aneurysmal sac is rare beyond 1 week after procedure and mostly reflecting clinically insignificant air hosted during stent graft deployment (7). Perigraft air observed 1 month after the procedure is deliberated pathognomonic for aortic graft infection (8), and CT scan is not essential for routine examination in the absence of other clinical signs of infection within first month after procedure (6). Although the air bubble established in the aneurysmal sac might be a coincidental finding, we believe that graft infection is often fatal if left untreated. Also, our patient had abdominal pain and his infection markers were substantially high. Treatment options for endograft infection include conservative therapy with antibiotics and surgical removal of the graft. We initiated antibiotic therapy and patient's complaint; infection markers and CT findings were resolved promptly.

In conclusion, thorough knowledge of the potential complications of EVAR, accurate radiological imaging, early diagnosis, and appropriate treatment are mandatory to reduce morbidity or even mortality due to these complications. In this context, air bubble within the excluded aneurysmal sac in the early post-procedure period might be an important finding of infection, thereby; it should be monitored closely.

\section{Acknowledgements}

The patient was evaluated and treated by MC and UCY. Radiologic data was analyzed by UB. Manuscript was prepared by MC. All the authors have participated in the drafting of the manuscript.

\section{Conflict of Interest}

No conflict of interest exists related to this article.

\section{References}

1. EVAR trial participants. Endovascular aneurysm repair versus open repair in patients with abdominal aortic an- eurysm (EVAR trial 1): randomised controlled trial. Lancet. 2005;365(9478):2179-2186.

2. Maleux G, Koolen M, Heye S. Complications after endovascular aneurysm repair. Semin Intervent Radiol 2009;26(1):3-9.

3. Tsoumakidou G, Brountzos E. Detection of complications after aortic stent grafting. European Cardiology 2010; 6(2):83-87.

4. Ducasse E, Calisti A, Speziale F, et al. Aortoiliac stent graft infection: current problems and management. Ann Vasc Surg 2004;18(5):521-526.

5. Capoccia L, Speziale F, Menna D, et al. Preliminary Results from a National Enquiry of Infection in Abdominal Aortic Endovascular Repair (Registry of Infection in EVAR--R.I.EVAR). Ann Vasc Surg 2016:198-204.

6. Kutlu R, Nisanoğlu V. Air within the aneurysm sac following endovascular management of abdominal aortic aneurysm in a patient with acute pancreatitis. Diagn Interv Radiol 2009;15(2):153-156.

7. Sawhney R, Kerlan RK, Wall SD, et al. Analysis of initial CT findings after endovascular repair of abdominal aortic aneurysm. Radiology 2001;220(1):157-160.

8. Orton DF, LeVeen RF, Saigh JA, et al. Aortic prosthetic graft infections: radiologic manifestations and implications for management. Radiographics 2000;20(4):977963. 